\title{
Article \\ Uniform Dichotomy Concepts for Discrete-Time Skew
Evolution Cocycles in Banach Spaces
}

\author{
Ariana Găină ${ }^{1,+} \mathbb{C}$, Mihail Megan ${ }^{1,2, *,+(\mathbb{D})}$ and Carmen Florinela Popa ${ }^{1,+(\mathbb{C}}$ \\ 1 Department of Mathematics, Faculty of Mathematics and Computer Science, West University of Timişoara, \\ 300223 Timişoara, Romania; ariana.gaina@e-uvt.ro (A.G.); carmen.popa95@e-uvt.ro (C.F.P.) \\ 2 Academy of Romanian Scientists, 050094 Bucharest, Romania \\ * Correspondence: mihail.megan@e-uvt.ro \\ + These authors contributed equally to this work.
}

check for

updates

Citation: Găină, A.; Megan, M.; Popa, C.F. Uniform Dichotomy Concepts for Discrete-Time Skew Evolution Cocycles in Banach Spaces. Mathematics 2021, 9, 2177.

https://doi.org/10.3390/math9172177

Academic Editor: Ioannis G. Stratis

Received: 12 July 2021

Accepted: 3 September 2021

Published: 6 September 2021

Publisher's Note: MDPI stays neutral with regard to jurisdictional claims in published maps and institutional affiliations.

\begin{abstract}
In the present paper, we consider the problem of dichotomic behaviors of dynamical systems described by discrete-time skew evolution cocycles in Banach spaces. We study two concepts of uniform dichotomy: uniform exponential dichotomy and uniform polynomial dichotomy. Some characterizations of these notions and connections between these concepts are given.
\end{abstract}

Keywords: uniform exponential dichotomy; uniform polynomial dichotomy; discrete-time skew evolution cocycles

MSC: 34D05; 34D09

\section{Introduction}

Recently, there has been a big interest in the qualitative theory of asymptotic behaviors of dynamical systems in infinite dimensional spaces, both in terms of continuous systems and of discrete systems. We consider not only the restrictive type of dichotomic behavior like uniform exponential dichotomy, but the more general type of behavior like uniform polynomial dichotomy.

The property of uniform exponential dichotomy introduced by Perron in 1930 [1], has been intensively studied, in this sense we mention the monographs of W. A. Coppel [2], J.L. Daleckii and M.G. Krein [3], J.L. Massera and J.J. Shäffer [4]. For practical examples for dichotomy concepts, we refer to these works and references. The case of uniform polynomial behaviors was studied by R. Barreira and C. Valls [5].

The notion of evolution cocycle considered as a generalization of the evolution operators characterizes the evolution of the systems described by differential equations with variable coefficients of the form $x^{\prime}(t)=A(t) x(t)$ and was introduced by M. Megan, C. Stoica and L. Buliga in the continuous case in [6] and by M. Megan and C. Stoica in [7] in the discrete case.

The study of the asymptotic behaviors of evolution cocycles was developed in the works of P. V. Hai [8], D. Dragicevic and C. Preda [9], M. Megan, A.L. Sasu and B. Sasu [10], as well as in the works of M. A. Tomescu [11], D. Borlea [12,13] and C.L.Mihiţ [14,15]. Furthermore, in the papers [16,17], different concepts of dichotomy for evolution cocycles were presented. The purpose of this article is to give some characterizations for uniform exponential dichotomy and uniform polynomial dichotomy of discrete-time skew evolution cocycles in Banach spaces.

The paper is organized as follows. In Section 2, we introduce the definitions and examples for discrete-time skew evolution cocycles, uniform exponential dichotomy, uniform polynomial dichotomy, uniform exponential growth and uniform polynomial growth. Connections between these concepts are emphasized. Some illustrating counterexamples are given. In Sections 3 and 4, we present the main results of this paper, where we firstly 
prove some characterizations of uniform polynomial dichotomy and secondly prove some characterizations of uniform exponential dichotomy. The conclusions and open problems are presented in the final section, Section 4.

\section{Preliminaries}

Let $X$ be a metric space, $V$ a Banach space and $\mathcal{B}(V)$ the Banach space of all bounded linear operators acting on $V$. We denote by

$$
\begin{gathered}
\Delta=\left\{(m, n) \in \mathbb{N}^{2}: m \geq n\right\} \\
T=\left\{(m, n, p) \in \mathbb{N}^{3}: m \geq n \geq p\right\}
\end{gathered}
$$

Definition 1. A mapping $\varphi: \Delta \times X \rightarrow X$ is called a discrete evolution semiflow on $X$ if the following conditions hold:

(es $\left.s_{1}\right) \quad \varphi(n, n, x)=x$, for all $(n, x) \in \mathbb{N} \times X$;

(es $) \quad \varphi(m, n, \varphi(n, p, x))=\varphi(m, p, x)$, for all $(m, n, p, x) \in T \times X$.

Definition 2. Let $\varphi: \Delta \times X \rightarrow X$ be a discrete evolution semiflow on $X$. An application $\Phi: \Delta \times X \rightarrow \mathcal{B}(V)$ is called discrete skew-evolution semiflow on $X \times V$ over $\varphi$ if:

(ses 1$) \quad \Phi(n, n, x)=I$ (the identity operator on $X)$, for all $(n, x) \in \mathbb{N} \times X$;

$\left(\right.$ ses $\left._{2}\right) \quad \Phi(m, n, \varphi(n, p, x)) \Phi(n, p, x)=\Phi(m, p, x)$, for all $(m, n, p, x) \in T \times X$.

If $\Phi$ is a discrete skew-evolution semiflow over the discrete evolution semiflow $\varphi$, then the pair $C=(\Phi, \varphi)$ is called a discrete-time skew evolution cocycle.

In the following three examples, we present some discrete-time skew evolution cocycles.

Example 1. For $X=\mathbb{N}$, the map $\varphi: \Delta \times X \rightarrow X$ defined by

$$
\varphi(m, n, x)=m-n+x
$$

is a discrete evolution semiflow on $X$.

Let $A_{n} \in \mathcal{B}(V), A: \Delta \rightarrow \mathcal{B}(V)$ be defined by

$$
A(m, n)=\left\{\begin{array}{r}
A_{m-1} \ldots A_{n}, m>n \\
I, m=n
\end{array}\right.
$$

and $\Phi: \Delta \times X \rightarrow \mathcal{B}(V)$ be defined by

$$
\Phi(m, n, x)=A(m-n+x, x)=A(\varphi(m, n, x), x) .
$$

We observe that $A(n, n)=I$ and $A(m, p)=A(m, n) A(n, p)$, for all $(m, n, p) \in T$.

Then, $\Phi$ is a discrete skew-evolution semiflow on $X \times V$ over the discrete evolution semiflow $\varphi$.

Example 2. Let $\varphi: \Delta \times X \rightarrow X$ be a discrete evolution semiflow on $X, A_{n}: X \rightarrow \mathcal{B}(V)$ and $\Phi: \Delta \times X \rightarrow \mathcal{B}(V)$ be defined by

$\Phi(m, n, x)=\left\{\begin{aligned} A_{m-1}(\varphi(m-1, n, x)) \ldots A_{n+1}(\varphi(n+1, n, x)), m & >n \\ I, m & =n\end{aligned}\right.$

Then, $\Phi$ is a discrete skew-evolution semiflow on $X \times V$ over discrete evolution semiflow $\varphi$.

Definition 3. The mapping $P: \mathbb{N} \times X \rightarrow \mathcal{B}(V)$ is called family of projectors if

$$
P^{2}(n, x)=P(n, x)
$$

for all $(n, x) \in \mathbb{N} \times X$. 
Remark 1. If $P: \mathbb{N} \times X \rightarrow \mathcal{B}(V)$ is a family of projectors, then the family of projectors $Q: \mathbb{N} \times$ $X \rightarrow \mathcal{B}(V)$ defined by $Q(n, x)=I-P(n, x)$ is called the complementary family of projectors $P$.

Definition 4. The family of projectors $P: \mathbb{N} \times X \rightarrow \mathcal{B}(V)$ is said to be invariant to discrete-time skew-evolution cocycle $C=(\Phi, \varphi)$ if

$$
\Phi(m, n, x) P(n, x)=P(m, \varphi(m, n, x)) \Phi(m, n, x),
$$

for all $(m, n, x) \in \Delta \times X$.

Example 3. Let $V=\mathbb{R}^{2}, P(n, x)\left(v_{1}, v_{2}\right)=\left(v_{1}, 0\right), Q(n, x)\left(v_{1}, v_{2}\right)=\left(0, v_{2}\right)$ and $\Phi: \Delta \times X \rightarrow \mathcal{B}(V)$ defined by

$$
\Phi(m, n, x)=e^{-(m-n)} P(n, x)+e^{m-n} Q(n, x) .
$$

Then, $P$ is invariant to discrete-time skew-evolution cocycle $C=(\Phi, \varphi)$, for all $\varphi$.

Definition 5. The pair $(C, P)$ is called uniformly exponentially dichotomic (u.e.d.) if there are $N \geq 1, v>0$ with:

$\left(\right.$ ued $\left._{1}\right) \quad\|\Phi(m, n, x) P(n, x) v\| \leq N e^{-v(m-n)}\|P(n, x) v\| ;$

$\left(\right.$ ued $\left._{2}\right) \quad e^{v(m-n)}\|Q(n, x) v\| \leq N\|\Phi(m, n, x) Q(n, x) v\|$,

for all $(m, n, x, v) \in \Delta \times X \times V$.

Remark 2. In Definition 5, it can be supposed that $v \in(0,1)$.

Definition 6. The pair $(C, P)$ has uniform exponential growth (u.e.g.) if there are $M>1$, $\omega>0$ with:

(ueg 1$) \quad\|\Phi(m, n, x) P(n, x) v\| \leq M e^{\omega(m-n)}\|P(n, x) v\| ;$

$\left(\right.$ ueg $\left._{2}\right) \quad e^{-\omega(m-n)}\|Q(n, x) v\| \leq M\|\Phi(m, n, x) Q(n, x) v\|$,

for all $(m, n, x, v) \in \Delta \times X \times V$.

$$
\text { Let } \Delta_{1}=\left\{(m, n) \in \mathbb{N}^{2} \mid m \geq n \geq 1\right\} \text {. }
$$

Remark 3. The pair $(C, P)$ has uniform exponential growth if and only if $\left(u_{e} g_{1}\right)$ and $\left(u e g_{2}\right)$ take place for $\omega>1$ and $(m, n, x, v) \in \Delta_{1} \times X \times V$.

Remark 4. We suppose that the pair $(C, P)$ has uniform exponential growth. The pair $(C, P)$ is uniformly exponentially dichotomic if and only if $\left(\right.$ ued $\left._{1}\right)$ and $\left(\right.$ ued $\left._{2}\right)$ take place for $(m, n, x, v) \in$ $\Delta_{1} \times X \times V$.

Definition 7. The pair $(C, P)$ is called uniformly polynomially dichotomic (u.p.d.) if there are $N \geq 1, v>0$ with:

$\left(\right.$ upd $\left._{1}\right) \quad(m+1)^{v}\|\Phi(m, n, x) P(n, x) v\| \leq N(n+1)^{v}\|P(n, x) v\| ;$

$\left(u_{p d}\right) \quad(m+1)^{v}\|Q(n, x) v\| \leq N(n+1)^{v}\|\Phi(m, n, x) Q(n, x) v\|$,

for all $(m, n, x, v) \in \Delta \times X \times V$.

Definition 8. The pair $(C, P)$ has uniform polynomial growth (u.p.g.) if there are $M>1$, $\omega>0$ with:

$\left(u_{p g}\right) \quad(n+1)^{\omega}\|\Phi(m, n, x) P(n, x) v\| \leq M(m+1)^{\omega}\|P(n, x) v\| ;$

$\left(u_{p g}\right) \quad(n+1)^{\omega}\|Q(n, x) v\| \leq M(m+1)^{\omega}\|\Phi(m, n, x) Q(n, x) v\|$,

for all $(m, n, x, v) \in \Delta \times X \times V$. 
Remark 5. The implications between the concepts of dichotomy and the concepts of growth are given by the diagram

$$
\begin{array}{ccc}
\text { u.e.d. } & \Rightarrow & \text { u.p.d. } \\
\Downarrow & & \Downarrow \\
\text { u.e.g., } & \Leftarrow & \text { u.p.g. }
\end{array}
$$

In the following four examples, we prove that the converse implications for the previous Remark are not true.

Example 4. Let $V=\mathbb{R}^{2}$ with ||$\left(v_{1}, v_{2}\right)||=\left|v_{1}\right|+\left|v_{2}\right|$ and $\varphi: \Delta \times X \rightarrow X$ be a discrete evolution semiflow on $X$. Then, $\Phi: \Delta \times X \rightarrow \mathcal{B}(V)$ defined by

$$
\Phi(m, n, x)\left(v_{1}, v_{2}\right)=\left(\frac{n+1}{m+1} v_{1}, \frac{m+1}{n+1} v_{2}\right),
$$

is a discrete evolution semiflow over $\varphi$, for all $(m, n) \in \Delta, x \in X,\left(v_{1}, v_{2}\right) \in \mathbb{R}^{2}$.

Let $P: \mathbb{N} \times X \rightarrow \mathcal{B}(V)$ be the family of projectors defined by

$$
P(n, x)\left(v_{1}, v_{2}\right)=\left(v_{1}, 0\right),
$$

for all $(n, x) \in \mathbb{N} \times X$ and $\left(v_{1}, v_{2}\right) \in \mathbb{R}^{2}$.

The complementary family of projectors $Q: \mathbb{N} \times X \rightarrow \mathcal{B}(V)$ is defined by

$$
Q(n, x)\left(v_{1}, v_{2}\right)=\left(0, v_{2}\right),
$$

for all $(n, x) \in \mathbb{N} \times X$ and $\left(v_{1}, v_{2}\right) \in \mathbb{R}^{2}$.

Then, the discrete-time skew evolution cocycle $C=(\Phi, \varphi)$ satisfies Definition 7 for $v=1$ and for all $N>1$. It results that $(C, P)$ is uniformly polynomially dichotomic.

If we assume that the pair $(C, P)$ is uniformly exponentially dichotomic, we would obtain that there are $N>1, v>0$ with

$$
\|\Phi(m, n, x) P(n, x) v\|\left\|\leq N e^{-v(m-n)}\right\| P(n, x) v \|
$$

It results that

$$
e^{v(m-n)} \frac{n+1}{m+1} \leq N
$$

For $m=2 n+1$ and $n \in \mathbb{N}$, we obtain

$$
e^{v(n+1)} \frac{1}{2} \leq N
$$

where, for $n \rightarrow+\infty$, we have contradiction.

So we have that the pair $(C, P)$ is not uniformly exponentially dichotomic.

Example 5. For $V=\mathbb{R}^{2}$ with the same norm as in Example $4, X$ arbitrary and the discrete skew-evolution semiflow $\Phi: \Delta \times X \rightarrow \mathcal{B}(V)$ over the discrete evolution semiflow $\varphi$ defined by

$$
\Phi(m, n, x)\left(v_{1}, v_{2}\right)=\left(\frac{m+1}{n+1} v_{1}, \frac{n+1}{m+1} v_{2}\right),
$$

for all $(m, n) \in \Delta, x \in X,\left(v_{1}, v_{2}\right) \in \mathbb{R}^{2}$.

The family of projectors $P$ and $Q$ is defined as in Example 4.

Then, $\Phi$ is discrete skew-evolution semiflow over all discrete evolution semiflow $\varphi: \Delta \times X \rightarrow X$ and $C=(\Phi, \varphi)$ is a discrete-time skew evolution cocycle.

So, for $\omega=1$ and for all $M>1,(C, P)$ verifies Definition 8. It results that $(C, P)$ has uniform polynomial growth. such as

If the pair $(C, P)$ would be uniformly polynomially dichotomic, we would obtain $N>1, v>0$ 


$$
\|\Phi(m, n, x) P(n, x) v\|\left\|N\left(\frac{n+1}{m+1}\right)^{v}\right\| P(n, x) v \|
$$

Therefore,

$$
\left(\frac{m+1}{n+1}\right)^{v+1} \leq N
$$

For $n=0$ and $m \in \mathbb{N}$, we have

$$
(m+1)^{v+1} \leq N
$$

where, for $m \rightarrow+\infty$, we have contradiction.

So the pair $(C, P)$ is not uniformly polynomially dichotomic.

Example 6. If we consider $V$ and $X$ as in Example 4, $\Phi: \Delta \times X \rightarrow \mathcal{B}(V)$ the discrete skewevolution semiflow over the discrete evolution semiflow $\varphi$ defined by

$$
\Phi(m, n, x)\left(v_{1}, v_{2}\right)=\left(e^{m-n} v_{1}, e^{n-m} v_{2}\right),
$$

for all $(m, n) \in \Delta, x \in X,\left(v_{1}, v_{2}\right) \in \mathbb{R}^{2}$ and the family of projectors $P$ and $Q$ is defined as in Example 4, then $\Phi$ is a discrete skew-evolution semiflow over all discrete evolution semiflow $\varphi: \Delta \times X \rightarrow X$ and $C=(\Phi, \varphi)$ is a discrete-time skew evolution cocycle.

The pair $(C, P)$ has uniform exponential growth as it satisfies Definition 6 for $\omega=1$ and for all $M>1$.

If we assume that the pair $(C, P)$ is uniformly exponentially dichotomic, we would obtain that there are $N>1, v>0$ with

$$
\|\Phi(m, n, x) P(n, x) v \mid\| \leq N e^{-v(m-n)}\|P(n, x) v\|
$$

So

$$
e^{m-n} \leq N e^{-v(m-n)}
$$

For $n=0$ and $m \in \mathbb{N}$, we have

$$
e^{m(v+1)} \leq N
$$

We have contradiction for $m \rightarrow+\infty$.

Therefore, the pair $(C, P)$ is not uniform exponentially dichotomic.

Example 7. Let $V, X, \Phi, P$ and $Q$ be defined as is Example 6.

So $\Phi$ is a discrete skew-evolution semiflow over all discrete evolution semiflow $\varphi: \Delta \times X \rightarrow X$, $C=(\Phi, \varphi)$ is a discrete-time skew evolution cocycle and $(C, P)$ has uniform exponential growth, for $\omega=1$ and for all $M>1$.

If we suppose that the pair $(C, P)$ has uniform polynomial growth, then we would have that there are $M>1, \omega>0$ with

$$
\|\Phi(m, n, x) P(n, x) v \mid\| \leq M\left(\frac{m+1}{n+1}\right)^{\omega}\|P(n, x) v\|
$$

It follows that

$$
e^{m-n} \leq M\left(\frac{m+1}{n+1}\right)^{\omega}
$$

Considering $m=2 n+1$ and $n \in \mathbb{N}$, we obtain

$$
e^{n+1} \frac{1}{2^{\omega}} \leq N
$$


So we have a contradiction for $n \rightarrow+\infty$.

Therefore, the pair $(C, P)$ does not have uniform polynomial growth.

\section{Characterizations for Uniform Polynomial Dichotomy}

Let $\Phi: \Delta \times X \rightarrow \mathcal{B}(V)$ be a discrete skew-evolution semiflow over the discrete evolution semiflow $\varphi: \Delta \times X \rightarrow X$ and $P: \mathbb{N} \times X \rightarrow \mathcal{B}(V)$ an invariant family of projectors.

Theorem 1. We suppose that $(C, P)$ has uniform polynomial growth. Then, the pair $(C, P)$ is uniformly polynomially dichotomic if and only if there exist $c \in(0,1), r \in \mathbb{N}^{*}, r>1$ with:

$\left(u p H_{1}\right) \quad\|\Phi(r n, n, x) P(n, x) v\| \leq c\|P(n, x) v\|$

$\left(u p H_{2}\right) \quad\|Q(n, x) v\| \leq c\|\Phi(r n, n, x) Q(n, x) v\|$,

for all $(n, x, v) \in \mathbb{N} \times X \times V$.

Proof. Necessity. Let $r=1+\left[N^{\frac{1}{v}}\right]$ and $c=\frac{N}{r^{v}}$.

From $\left(u p d_{1}\right)$ we have that

$$
\|\Phi(r n, n, x) P(n, x) v\| \leq N\left(\frac{n+1}{r n+1}\right)^{v}\|P(n, x) v\| \leq c\|P(n, x) v\| .
$$

From $\left(u p d_{2}\right)$ we have that

$$
c\|\Phi(r n, n, x) Q(n, x) v\| \geq\|Q(n, x) v\| .
$$

Sufficiency. Let $p=\left[\frac{\ln m-\ln n}{\ln r}\right], N=M r^{\omega}$ and $v=-\frac{\ln c}{\ln r}$.

$$
\begin{aligned}
& \|\Phi(m, n, x) P(n, x) v\|= \\
= & \left\|\Phi\left(m, n r^{p}, \varphi\left(n r^{p}, n, x\right)\right) P\left(n r^{p}, \varphi\left(n r^{p}, n, x\right)\right) \Phi\left(n r^{p}, n, x\right) P(n, x) v\right\| \\
\leq & M\left(\frac{m+1}{n r^{p}+1}\right)^{\omega}\left\|\Phi\left(n r^{p}, n, x\right) P(n, x) v\right\| \\
\leq & M\left(\frac{m+1}{n r^{p}+1}\right)^{\omega} \| \Phi\left(n r^{p}, n r^{p-1}, \varphi\left(n r^{p-1}, n, x\right)\right) P\left(n r^{p-1}, \varphi\left(n r^{p-1}, n, x\right)\right) \\
& \Phi\left(n r^{p-1}, n, x\right) P(n, x) v \| \\
\leq & M\left(\frac{m+1}{n r^{p}+1}\right)^{\omega} c\left\|\Phi\left(n r^{p-1}, n, x\right) P(n, x) v\right\| \leq \ldots \leq \\
\leq & M\left(\frac{m+1}{n r^{p}+1}\right)^{\omega} c^{p}\|P(n, x) v\| \\
\leq & N\left(\frac{n+1}{m+1}\right)^{v}\|P(n, x) v\| .
\end{aligned}
$$


Similarly, we have

$$
\begin{aligned}
& \mathbf{N}\|\Phi(m, n, x) Q(n, x) v\|= \\
= & \mathbf{N}\left\|\Phi\left(m, n r^{p}, \varphi\left(n r^{p}, n, x\right)\right) Q\left(n r^{p}, \varphi\left(n r^{p}, n, x\right)\right) \Phi\left(n r^{p}, n, x\right) Q(n, x) v\right\| \\
\geq & \frac{N}{M}\left(\frac{n r^{p}+1}{m+1}\right)^{\omega}\left\|\Phi\left(n r^{p}, n, x\right) Q(n, x) v\right\| \\
\geq & \frac{N}{M}\left(\frac{n r^{p}+1}{m+1}\right)^{\omega} \| \Phi\left(n r^{p}, n r^{p-1}, \varphi\left(n r^{p-1}, n, x\right)\right) Q\left(n r^{p-1}, \varphi\left(n r^{p-1}, n, x\right)\right) \\
& \Phi\left(n r^{p-1}, n, x\right) Q(n, x) v \| \\
\geq & \frac{N}{M}\left(\frac{n r^{p}+1}{m+1}\right)^{\omega} \frac{1}{c}\left\|\Phi\left(n r^{p-1}, n, x\right) Q(n, x) v\right\| \geq \ldots \geq \\
\geq & \frac{N}{M}\left(\frac{n r^{p}+1}{m+1}\right)^{\omega} \frac{1}{c^{p}}\|Q(n, x) v\| \\
\geq & \left(\frac{m+1}{n+1}\right)^{v}\|Q(n, x) v\| .
\end{aligned}
$$

Remark 6. The previous theorem is a generalization to the uniform polynomial dichotomy of the discrete-time skew-evolution cocycles in Banach spaces of a result from the theory of uniform polynomial dichotomy obtained by C.L. Mihiţ and M. Lăpădat [15] for the case of skew-evolution semiflows on the half-line and by R. Boruga and M.Megan [18] for evolution operators.

The next theorem is a logarithmic criteria for the uniform polynomial dichotomy for discrete-time skew evolution cocycles in Banach space.

Theorem 2. We suppose that $(C, P)$ has uniform polynomial growth. Then, the pair $(C, P)$ is uniformly polynomially dichotomic if and only if there exist $L>1$ with:

$\left(u p l_{1}\right) \quad\|\Phi(m, n, x) P(n, x) v\| \ln \frac{m+1}{n+1} \leq L\|P(n, x) v\|$

$\left(u p l_{2}\right) \quad\|Q(n, x) v\| \ln \frac{m+1}{n+1} \leq L\|\Phi(m, n, x) Q(n, x) v\|$,

for all $(m, n, x, v) \in \Delta \times X \times V$.

Proof. Necessity. Let $L=1+\frac{N}{v e}$.

From $\left(u p d_{1}\right)$ we have that

$$
\begin{aligned}
\|\Phi(m, n, x) P(n, x) v\| \ln \frac{m+1}{n+1} & \leq \frac{N}{v}\left(\frac{m+1}{n+1}\right)^{-v}\|P(n, x) v\| \ln \left(\frac{m+1}{n+1}\right)^{v} \\
& \leq \frac{N}{v e}\|P(n, x) v\| \\
& \leq L\|P(n, x) v\| .
\end{aligned}
$$

From $\left(u p d_{2}\right)$ we have that

$$
\begin{aligned}
\|Q(n, x) v\| \ln \frac{m+1}{n+1} & \leq \frac{N}{v}\left(\frac{n+1}{m+1}\right)^{v}\|\Phi(m, n, x) Q(n, x) v\| \ln \left(\frac{m+1}{n+1}\right)^{v} \\
& \leq \frac{N}{v e}\|\Phi(m, n, x) Q(n, x) v\| \\
& \leq L\|\Phi(m, n, x) Q(n, x) v\| .
\end{aligned}
$$

Sufficiency. Let $c=\left(\frac{1}{2}, 1\right)$ and $r=\left[e^{4 L}\right]$. 
By $\left(u p l_{1}\right)$, we have that

$$
\|\Phi(r n, n, x) P(n, x) v\| \leq \frac{L}{\ln \frac{n r+1}{n+1}}\|P(n, x) v\| \leq c\|P(n, x) v\|,
$$

which implies $\left(u p H_{1}\right)$.

By $\left(u p l_{2}\right)$, we have that

$$
\|\Phi(r n, n, x) Q(n, x) v\| \geq \frac{1}{L} \ln \frac{n r+1}{n+1}\|Q(n, x) v\| \geq 2\|Q(n, x) v\|,
$$

which implies $\left(\mathrm{upH}_{2}\right)$.

So we have $\|Q(n, x) v\| \leq c\|\Phi(r n, n, x) Q(n, x) v\|$, where $c=\frac{1}{2}$.

Remark 7. The particular case when $\Phi$ is an evolution operator, is considered in [18].

The majorization criteria for the uniform polynomial dichotomy for discrete-time skew evolution cocycles in Banach space is presented in the following theorem.

Theorem 3. If $(C, P)$ has uniform polynomial growth. Then, the pair $(C, P)$ is uniformly polynomially dichotomic if and only if there exist $M: \mathbb{R}_{+}^{*} \rightarrow(1, \infty)$ nondecreasing with $\lim _{n \rightarrow \infty} M(n)=\infty$ such that:

$\left(\right.$ upM $\left.M_{1}\right) \quad M\left(\frac{m+1}{n+1}\right)\|\Phi(m, n, x) P(n, x) v\| \leq\|P(n, x) v\|$

$\left(u_{p} M_{2}\right) \quad M\left(\frac{m+1}{n+1}\right)\|Q(n, x) v\| \leq\|\Phi(m, n, x) Q(n, x) v\|$

for all $(m, n, x, v) \in \Delta \times X \times V$.

Proof. Necessity. It follows from Theorem 2 for $M(n)=\frac{\ln n}{L}$.

Sufficiency. It follows from Theorem 1 for $c=\frac{1}{M(1)} \in(0,1)$.

Remark 8. The particular case for previous theorem when $\Phi$ is an evolution operator was considered by R. Boruga in [19].

\section{Characterizations for Uniform Exponential Dichotomy}

Let $\Phi: \Delta \times X \rightarrow \mathcal{B}(V)$ be a discrete skew-evolution semiflow over the discrete evolution semiflow $\varphi: \Delta \times X \rightarrow X$ and $P: \mathbb{N} \times X \rightarrow \mathcal{B}(V)$ an invariant family of projectors.

Theorem 4. We suppose that $(C, P)$ has uniform exponential growth. Then, the pair $(C, P)$ is uniformly exponentially dichotomic if and only if there exist $c \in(0,1), r \in \mathbb{N}^{*}, r>1$ with:

$\left(u e H_{1}\right) \quad\|\Phi(r+n, n, x) P(n, x) v\| \leq c\|P(n, x) v\|$

$\left(u e H_{2}\right) \quad\|Q(n, x) v\| \leq c\|\Phi(r+n, n, x) Q(n, x) v\|$,

for all $(n, x, v) \in \mathbb{N} \times X \times V$.

Proof. Necessity. Let $r=1+\left[\frac{\ln N}{v}\right]$, where [.] denotes the integer part and $c=N e^{-r v}$. From $\left(\right.$ ued $\left._{1}\right)$ we have that

$$
\|\Phi(r+n, n, x) P(n, x) v\| \leq N e^{-r v}\|P(n, x) v\|=c\|P(n, x) v\| .
$$

From $\left(\right.$ ued $\left._{2}\right)$ we have that

$$
c\|\Phi(r+n, n, x) Q(n, x) v\| \geq\|Q(n, x) v\| .
$$

Sufficiency. Let $p=\left[\frac{m-n}{r}\right], N=\frac{M e^{\omega r}}{c}$ and $v=-\frac{\ln c}{r}$. 


$$
\begin{aligned}
& \|\Phi(m, n, x) P(n, x) v\|= \\
= & \|\Phi(m, n+r p, \varphi(n+r p, n, x)) P(n+r p, \varphi(n+r p, n, x)) \Phi(n+r p, n, x) P(n, x) v\| \\
\leq & M e^{\omega(m-n-r p)}\|\Phi(n+r p, n, x) P(n, x) v\| \\
\leq & M e^{\omega r} \| \Phi(n+r p, n+r(p-1), \varphi(n+r(p-1), n, x)) P(n+r(p-1), \varphi(n+r(p-1), n, x)) \\
& \Phi(n+r(p-1), n, x) P(n, x) v \| \\
\leq & M e^{\omega r} c\|\Phi(n+r(p-1), n, x) P(n, x) v\| \leq \ldots \leq \\
\leq & M e^{\omega r} c^{p}\|P(n, x) v\| \\
= & \frac{M e^{\omega r}}{c} c^{p+1}\|P(n, x) v\| \\
= & N e^{-v(m-n)}\|P(n, x) v\| .
\end{aligned}
$$

Similarly, we have

$$
\begin{aligned}
N & \|\Phi(m, n, x) Q(n, x) v\|= \\
= & N\|\Phi(m, n+r p, \varphi(n+r p, n, x)) Q(n+r p, \varphi(n+r p, n, x)) \Phi(n+r p, n, x) Q(n, x) v\| \\
\geq & \frac{N}{M} e^{-\omega(m-n-r p)}\|\Phi(n+r p, n, x) Q(n, x) v\| \\
\geq & \frac{N}{M} e^{-\omega r} \| \Phi(n+r p, n+r(p-1), \varphi(n+r(p-1), n, x)) Q(n+r(p-1), \varphi(n+r(p-1), n, x)) \\
& \Phi(n+r(p-1), n, x) Q(n, x) v \| \\
\geq & \frac{N}{M c} e^{-\omega r}\|\Phi(n+r(p-1), n, x) Q(n, x) v\| \geq \ldots \geq \\
\geq & \frac{N}{M c^{p}} e^{-\omega r}\|Q(n, x) v\| \\
\geq & e^{v(m-n)}\|Q(n, x) v\| .
\end{aligned}
$$

Remark 9. The Theorem 4 is a generalization of some results presented by C. Stoica in [20].

In what follows, the logarithmic criteria for the uniform exponential dichotomy for discrete-time skew evolution cocycles in Banach space is proved.

Theorem 5. We suppose that $(C, P)$ has uniform exponential growth. Then, the pair $(C, P)$ is uniformly exponentially dichotomic if and only if there is $L>1$ with:

$\left(\right.$ uel $\left._{1}\right) \quad(m-n)\|\Phi(m, n, x) P(n, x) v\| \leq L\|P(n, x) v\|$

$\left(\right.$ uel $\left._{2}\right) \quad(m-n)\|Q(n, x) v\| \leq L\|\Phi(m, n, x) Q(n, x) v\|$

for all $(m, n, x, v) \in \Delta \times X \times V$.

Proof. Necessity. Let $L=1+\frac{N}{v e}$.

From $\left(u e d_{1}\right)$ we have that

$$
\begin{aligned}
(m-n)\|\Phi(m, n, x) P(n, x) v\| & \leq(m-n) N e^{-v(m-n)}\|P(n, x) v\| \\
& \leq \frac{N}{v e}\|P(n, x) v\| \\
& \leq L\|P(n, x) v\| .
\end{aligned}
$$


From $\left(\right.$ ued $\left._{2}\right)$ we have that

$$
\begin{aligned}
(m-n)\|Q(n, x) v\| & \leq(m-n) N e^{-v(m-n)}\|\Phi(m, n, x) Q(n, x) v\| \\
& \leq \frac{N}{v e}\|\Phi(m, n, x) Q(n, x) v\| \\
& \leq L\|\Phi(m, n, x) Q(n, x) v\| .
\end{aligned}
$$

Sufficiency. Let $c=\frac{L}{r}$ and $r=L+1$.

By $\left(u e l_{1}\right)$, we have that

$$
\|\Phi(r+n, n, x) P(n, x) v\| \leq \frac{L}{r}\|P(n, x) v\|=c\|P(n, x) v\|,
$$

which implies $\left(u e H_{1}\right)$.

By $\left(u e l_{2}\right)$, we have that

$$
c\|\Phi(r+n, n, x) Q(n, x) v\|=\frac{L}{r}\|\Phi(r+n, n, x) Q(n, x) v\| \geq\|Q(n, x) v\|,
$$

which implies $\left(u e \mathrm{H}_{2}\right)$.

The next theorem presents a majorization criteria for the uniform exponential dichotomy for discrete-time skew evolution cocycles in Banach space.

Theorem 6. If $(C, P)$ has uniform exponential growth. Then, the pair $(C, P)$ is uniformly exponentially dichotomic if and only if there exist $M: \mathbb{R}_{+}^{*} \rightarrow(1, \infty)$ nondecreasing with $\lim _{n \rightarrow \infty} M(n)=\infty$ such that:

$\left(\right.$ ueM $\left.M_{1}\right) \quad M(m-n)\|\Phi(m, n, x) P(n, x) v\| \leq\|P(n, x) v\|$

$\left(u e M_{2}\right) \quad M(m-n)\|Q(n, x) v\| \leq\|\Phi(m, n, x) Q(n, x) v\|$

for all $(m, n, x, v) \in \Delta \times X \times V$.

Proof. Necessity. It follows from Theorem 5 for $M(n)=\frac{n}{L}$.

Sufficiency. It follows from Theorem 4 for $c=\frac{1}{M(1)} \in(0,1)$.

Remark 10. The particular case for Theorem 6, when $\Phi$ is a skew-evolution semiflow on Banach spaces, was considered by C. Stoica in [20].

\section{Conclusions}

In this paper, we obtained three types of characterization for uniform exponential dichotomy and uniform polynomial dichotomy of dynamical systems described by discretetime skew evolution cocycles in Banach space. We gave the connection between these concepts, and also examples and counterexamples. As open problems, we have in mind generalization of these results for the nonuniform case, as well as for the case of behaviors with growth rates in which exponential and polynomial dichotomies appear as particular cases and much more, variants of these results for the concept of trichotomy.

Author Contributions: Conceptualization, A.G., M.M. and C.F.P.; Investigation, A.G., M.M. and C.F.P.; Methodology, A.G., M.M. and C.F.P.; Supervision, M.M.; Writing - original draft, A.G., M.M. and C.F.P.; Writing - review \& editing, M.M. All authors (A.G., M.M. and C.F.P.) contributed equally in the development of this work. All authors have read and agreed to the published version of the manuscript.

Funding: This research received no external funding.

Institutional Review Board Statement: Not applicable.

Informed Consent Statement: Not applicable. 
Data Availability Statement: Not applicable.

Acknowledgments: The authors wish to express special thanks to the reviewers for their helpful suggestions, which led to the improvement of this paper.

Conflicts of Interest: The authors declare no conflict of interest.

\section{References}

1. Perron, O. Die stabilitatsfrage bei differential gleichungen. Math. Z. 1930, 32, 703-728. [CrossRef]

2. Coppel, W.A. Dichotomies in stability theory. In Lecture Notes in Math. No. 629; Springer: Berlin, Germany; New York, NY, USA, 1978.

3. Daleckii, J.L. ; Krein, M.G. Stability of Solutions of Differential Equations in Banach Spaces; Translations of Mathematical Monographs; American Mathematical Society: Providence, RI, USA, 1974; Volume 43.

4. Massera, J.L.; Schäffer, J.J. Linear Differential Equations and Function Spaces; Academic Press: New York, NY, USA, 1966.

5. Barreira, L.; Valls, C. Polynomial growth rates. Nonlinear Anal. 2009, 71, 5208-5219. [CrossRef]

6. Megan, M.; Stoica, C.; Buliga, L. On asymptotic behaviors for linear skew-evolution semiflows in Banach spaces. Carpathian J. Math. 2007, 23, 117-125.

7. Megan, M.; Stoica, C. Discrete asymptotic behaviors for skew-evolution semiflows on Banach spaces. Carpathian J. Math. 2008, 24, 348-355.

8. Hai, P.V. Polynomial Stability and Polynomial Instability for Skew-Evolution Semiflows. Results Math. 2019, 74, 175. [CrossRef]

9. Dragičević, D.; Preda, C. Lyapunov Type Theorems for Exponential Stability of Linear Skew-Product Three-Parameter Semiflows with Discrete Time. Axioms 2020, 9, 47. [CrossRef]

10. Megan, M.; Sasu, A.L.; Sasu, B. Exponential stability and exponential instability for linear skew-product flows. Math. Bohem. 2004, 129, 225-243. [CrossRef]

11. Tomescu, M.A.; Megan, M.; Ceauşu, T. On Exponential Dichotomy of Variational Nonautonomous Difference Equations in Banach Spaces, Recent Advances in Applied and Theoretical Mathematics. In Proceedings of the 18th WSEAS International Conference on Applied Mathematics, Budapest, Hungary, 10-12 December 2013; pp. 37-44.

12. Borlea, D. Some Concepts of Uniform Exponential Dichotomy for Skew-Evolution Semiflows in Banach Spaces. Theory Appl. Math. Comput. Sci. 2016, 6, 69-76.

13. Patrascu Borlea, D. Uniform Polynomial Dichotomy for Linear Discrete-time Systems. Electron. Notes Discret. Math. 2018, 67, 79-83. [CrossRef]

14. Mihit, C.L.; Megan, M. On uniform exponential dichotomy of skew-evolution semiflows in Hilbert spaces. In Proceedings of the International Symposium Research and Education in an Innovation Era, Arad, Romania, 8-10 December 2016 ; pp. 30-37.

15. Mihiț, C.L.; Lăpădat, M. On uniform polynomial dichotomy of skew-evolution semiflows on the half-line. Bul. Ştiint. Univ. Politeh. Timiş. Ser. Mat. Fiz. 2017, 62 , 54-61.

16. Megan, M.; Stoica, C. Concepts of dichotomy for skew-evolution semiflows in Banach spaces. Ann. Acad. Rom. Sci. Ser. Math. Appl. 2010, 2, 125-140.

17. Popa, I.-L.; Megan, M.; Ceauşu, T. Exponential dichotomies for linear discrete-time systems in Banach spaces. Appl. Anal. Discrete Math. 2012, 6, 140-155. [CrossRef]

18. Boruga, R.; Megan, M. On uniform polynomial dichotomy in Banach spaces. Sci. Bull. Politeh. Univ. Timiş. $2018,63,77$.

19. Boruga, R. Majorization criteria for polynomial stability and instability of evolution operators. Sci. Bull. Politeh. Univ. Timiş. 2019, $64,78$.

20. Stoica, C. On Exponential Stability for Skew-Evolution Semiflows on Banach Spaces. arXiv 2008, arXiv:0804.1479v1. 